\title{
Induced pluripotent stem cells in infections
}

\author{
Anubha Singh, Deepak Kumar Singh, Usha Bhoria
}

\begin{abstract}
Regenerative medicine has an exciting role in the treatment of disorders and conditions where there is an irreversible damage to a tissue or an organ or in immunodeficient state. Stem cells which have the potential to differentiate into specific types of cells, offers a new concept of regenerative medicine to treat spectrum of diseases including infections. Infections are one of the cause of high morbidity during injuries and in immunodeficient states. Induced pluripotent stem cells (iPSCs) are generated by genetically reprogramming the adult cells to an embryonic stem cell (ESC)-like state by being forced to express genes and factors essential for maintaining the defining properties of ESC. In infections, eosinophils play an important role as they are potent source of cytotoxic mediators such as eosinophil cationic protein (ECP) and major basic proteins (MBP). These substances play an important role in immune response to infections caused by bacteria, viruses, parasites and in
\end{abstract}

Anubha Singh ${ }^{1}$ Deepak Kumar Singh², Usha Bhoria ${ }^{3}$

Affiliations: 'Senior Resident, Department of Laboratory Services, Employees' State Insurance Corporation Model Hospital, Ministry of Labor, Noida, Uttar Pradesh, India; 2Junior Specialist, Department of Laboratory Services, Employees' State Insurance Corporation Model Hospital, Ministry of Labor, Noida, Uttar Pradesh, India; ${ }^{3}$ Senior Specialist, Department of Laboratory Services, Employees' State Insurance Corporation Model Hospital, Ministry of labor, Noida, Uttar Pradesh, India.

Corresponding Author: Anubha Singh, Senior Resident, Department of Laboratory Services, Employees' State Insurance Corporation Model Hospital, Noida, Uttar Pradesh, India; E-mail: anubha.singh0111@gmail.com

Received: 27 March 2015

Accepted: 05 May 2015

Published: 19 May 2015 resistance to some forms of cancer. Strategies to enhance the intrinsic functional abilities of eosinophils by lineage specific differentiation of iPSC and enhanced expression of cytotoxic mediators by advanced genetic engineering approach provide important future application in such opportunistic infections.

Keywords: Induced pluripotent stem cell (iPSC), Reprogramming, Hematopoietic cells, Infections, Eosinophils

\section{How to cite this article}

Singh A, Singh DK, Bhoria U. Induced pluripotent stem cells in infections. Int $\mathrm{J}$ Blood Transfus Immunohematol 2015;5:14-18.

Article ID: 100017IJBTIAS2015

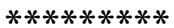

doi:10.5348/ijbti-2015-17-RA-3

\section{INTRODUCTION}

Stem cell transfusion is widely practiced clinical regimen in a patient with impaired immune system or with an immunodeficiency disease. Immunodeficiencies lead to increased risk of infections. The goal of stem cell transfusion is to provide functionally competent myeloid and lymphoid cells, which ensure survival and regain of normal health from opportunistic infections which is the main cause of immediate morbidity.

There have been several attempts to selectively engineer stem cells, Followed by their transfusion where they regenerate more number of specific immune cells for wide spectrum bactericidal actions [1]. The attempts 
involve prior treatment/culture of stem cells with specific set of cytokines to induce differentiation in the required direction of progenicity. This is an expensive method and unreliable in terms of quantum of highly effective bactericidal myeloid lineages, and therefore, in the recent past this has been over looked. In this regard, strategies to enhance functional abilities intrinsically in eosinophils by iPSCs approach can be proposed.

A proof-of-concept study using mouse iPSCs has clearly shown the potential of iPSCs for therapeutic cell replacement purposes [2]. This study used the iPSCs which were derived from humanized sickle cell mice, in which the mouse a-globin genes were replaced with the human sickle globin variants [2]. Through homologous recombination, the defective gene in the autologous iPSCs was repaired, and HSCs were generated from the genetically corrected iPSCs. Transplantation of the ex vivo-generated corrected HSCs rescued the sickle cell phenotype. This was an important novel approach which can be used for cure of genetic diseases. The purpose of this review is to discuss some selected studies done by authors where differentiation of iPSCs to lineagerestricted myeloid cells (eosinophils, important effector cells releasing cytotoxic mediators such as ECP, EDN, MBP, EPO), and role of iPSCs as cell-based therapy in various immune deficient conditions. We briefly discuss the role of eosinophils implicated in the pathogenesis of numerous biological responses, opportunistic infections including helminth infections and allergic diseases. Considering the potential of induced pluripotent stem cells (iPSCs) in differentiating into any type of cell in the human system we extrapolate the application of the iPSC technology to give rise to more lineage specific cells (eosinophil) in order to enhance the cytotoxic mediators such as eosinophil cationic protein in the system to combat the infections effectively. For this concept we discuss the selected studies done by authors.

\section{EOSINOPHILS AND THEIR ROLE IN INFECTIONS}

Myeloid and lymphoid cells are the caretakers of our immune system. Granules found in the eosinophil, which is a type of myeloid cell, are potent source of cytokines, basic proteins and other mediators. These substances play an important role in immune response to infections caused by bacteria [3], viruses [4], parasites [5-8] and in resistance to some forms of cancer [9].

Eosinophils are derived from pluripotent hematopoietic stem cells (HSCs) in the bone marrow. Hematopoietic stem cells (HSCs), capable of selfrenewal, are the precursors for all type of lineages of mature blood cells. These cells can be divided into short-term repopulating hematopoietic stem cells (STHSCs) and long-term repopulating hematopoietic stem cells (LT-HSCs).Short-term repopulating hematopoietic stem cells are known to differentiate into multipotent progenitor cells, which are capable of differentiation toward a subset of the hematopoietic lineage. Multipotent stem cells include the common myeloid and lymphoid precursor. Common myeloid progenitor cells, knownasthe granulocyte/erythrocyte/macrophage/ megakaryocyte colony-forming unit (CFU-GEMM), can differentiate toward the erythroid, megakaryocytic, and myelomonocytic lineage. It has been demonstrated that genes specific for erythroid, myeloid, or megakaryocytic lineages are transcribed in the common myeloid progenitors before commitment to a single lineage. On commitment to a single lineage, the genes which are specific for differentiation toward other lineages are downregulated [10].

Myeloid differentiation is regulated by a variety of cytokines, including erythropoietin (EPO), granulocytecolony-stimulating factor (G-CSF), thrombopoietin (TPO), interleukin-3 (IL-3), IL-5, granulocyte macrophage-colony-stimulating factor (GM-CSF), macrophage-colony-stimulating factor (M-CSF). IL-3 and GM-CSF are cytokines that regulate proliferation and survival during myeloid differentiation of various lineages, whereas EPO, TPO, G-CSF, M-CSF, and IL-5 are required for the final maturation of erythrocytes, megakaryocytes, platelets, neutrophils, monocytes, and eosinophils, respectively.

The myeloid lineage consists of eosinophils, mast cells, macrophages, basophils, neutrophils, and platelets. It mounts the body's first line of response, the innate immune response, when the body first encounters foreign pathogens. Pathogens that escape the first line of defense encounter the body's second line of defense, the humoral immune response, which is mounted by cells from the lymphoid lineage, including natural killer cells, $\mathrm{T}$ and $\mathrm{B}$ cells.

Eosinophils are multifunctional effector leukocytes implicated in the pathogenesis of numerous diseases and inflammatory processes. As the major effectors, eosinophils function in a variety of biological responses, opportunistic infections, allergic diseases, tumor immunity and helminth infections. The ribonucleases eosinophil-derived neurotoxin (EDN) and eosinophil cationic protein (ECP) are among the major secretory effector proteins of eosinophils in primates. The ECP and EDN are members of the RNase A superfamily, which play a crucial role in host defense against various pathogens as they are endowed with several biological activities. ECP has cytotoxic activity against gram positive and gram negative bacteria [3], viruses such as respiratory syncytial virus (RSV) [4], parasites such as trichinella spiralis, schistosoma mansoni, trypanosoma cruzi, leishmania donovani etc [5-8]. Helminthic infectionresults in enhancement of eosinophil numbers, directed migration and subsequent degranulation at the parasite surface, resulting in tegumental membrane damage and eventually killing the parasite. 


\section{INCREASING THE FUNCTIONAL ABILITIES OF EOSINOPHILS BY iPSCs APPROACH TO COMBAT INFECTIONS}

Damage to a tissue or an organ due to an injury or infection, is an irreversible process and in such conditions the role of regenerative medicine is important. Generation of cells and tissues that could be used for cell-based therapies is the most important application of human stem cells. The present approach of using donated organs and tissues to replace destroyed and degenerated tissues is limited by the availability of donors. Human stem cells, which can be directed to differentiate into any type of cells, provide the possibility of a renewable source of replaceable cells and tissues to treat a spectrum of infectious diseases.

Induced pluripotent stem cellsare generated by the genetic reprogramming of somatic cells by certain factors which are important in producing an embryonic stem cell-like state with similar features such as their morphology, gene expression, epigenetic modifications and differentiation potential [11]. Though some recent reports show that iPSC have more profound differences compared to embryonic stem cells (ESCs) than originally assumed [12]. Findings of genetic instability of iPSCs have also raised concerns about their potential use in the clinical setting. The iPSCs have been established to be useful tools for drug development and modelling of diseases with more advanced applications to be used in regenerative medicine. Breakthrough discovery of iPSC technology has created a powerful and innovative way to "de-differentiate" cells whose developmental fates had been assumed to be predetermined. An important point is that since the cells/tissues derived from iPSCs are patientspecific, they are nearly identical match to the cell donor and therefore, avoid rejection by the immune system. The iPSC and other types of pluripotent stem cells are important in building up strategies for reprogramming cells to repair damaged tissues and for immune boosting therapies, in the human body.

Main cause of morbidity in multiple scenarios is due to infections. It is important to combat infections in order to protect life by diminishing the chances of infections or secondary infections. Eosinophils secrete many cytotoxic granule proteins that have been identified as effector molecules responsible for many of the eosinophil actions in human system. The four major eosinophil granule proteins, major basic protein (MBP), eosinophil cationic protein (ECP), eosinophil derived neurotoxin (EDN) and eosinophil peroxidase have been shown to be involved in a number of eosinophil associated functions [13]. EDN possess antiviral activity against single stranded RNA viruses like respiratory syncytial virus, Hepatitis and HIV, whereas ECP and MBP exhibit antibacterial, antiparasitic and antiviral activities. ECP has also been shown to possess antiviral activity against single stranded RNA viruses like respiratory syncytial virus.
The induced pluripotent stem cells can have an important application in such opportunistic infections by

- Their enforced differentiation leading to myeloid (eosinophil) lineage thereby increasing the eosinophils to combat infections

- Genetic engineering approach to potentiate the functional abilities of eosinophils by enhancing its infection fighting mediators (increased expression of antimicrobials such as eosinophil cationic protein).

The primary challenge is to understand as to how the undifferentiated stem cells turn into differentiated cells that ultimately regenerate the tissues and organs. Turning genes on and off is central to this process. Predictably controlling cell proliferation and differentiation requires additional basic research on the molecular and genetic signals that regulate cell division and specialization. Studies to explore earlygenesis of eosinophils, especially in uncovering the mechanisms controlling the development of the initial innate immune system of human being in normal and diseased individuals is important.

\section{RESEARCH STUDIES}

There are several different strategies to direct ESC and iPSC towards the blood lineage [12]. Three different methods have been employed to induce pluripotent stem cells to become mesodermal cells: (1) the formation of embryoid bodies (EB), (2) the co-culturing of pluripotent stem cells with stromal layers, and (3) the culturing of pluripotent stem cells on extracellular matrix proteins. The former two methods are mainly utilized to differentiate iPSCs into hematopoietic progenitors. Erythrocyte, platelets and neutrophils have also been successfully differentiated from hESCs or iPSCs. However, generation of other blood cells has been more limited [12].

A study reported an efficient method for producing eosinophils from human embryonic and induced pluripotent stem cells (hESC/iPSCs). By a two-step induction, the authors first generated multipotential hematopoietic progenitors by co-culturing hESC/ iPSCs with mouse AGM-derived stromal cells for two weeks. Then, total co-culture cells were transferred into suspension culture favoring eosinophil development with addition of IL-3 and other factors (SCF, IL-6, TPO, Flt-3 ligand). The maturation of hESC/iPSC -derived eosinophils was shown in a time-dependent manner, first co-expressing eosinophil-and basophil-specific markers [eosinophil peroxidase (EPO), and 2D7, respectively], then the portion of eosinophil markers gradually increased while that of basophil markers decreased typically mimicking the development of eosinophils from human adult hematopoietic progenitors. By flow cytometric analysis, an eosinophil-specific surface marker, Siglec-8, was also expressed on these hESC/ iPSC-derived eosinophils in a time-dependent manner 
paralleling to those with EPO. The expression of eosinophil-specific granule cationic proteins (EPO, MBP, ECP, EDN) and IL-5 receptor mRNA was also detected by RT-PCR. Furthermore, transmission electron microscopy (TEM) observation confirmed the eosinophil property. Eosinophils derived from hiPSCs hold similar characteristics as those from hESCs [14].

\section{CONCLUSION}

It is only a decade since the concept of induced pluripotent stem cells came into existence. Initial efforts to culture iPSCs and further carrying out preclinical studies on murine and primates gave promising insights into their potential applications. The application of iPSCs in regenerative medicine gave hope to victims who are immunodeficient and those whose organs are damaged irreversibly. Most of the injuries and an immunodeficiency state cause morbidity due to opportunistic infections. Eosinophils are important effector cells as they contain plethora of cytotoxic mediators such as ECP, EDN, MBP, EPO and many more cytokines. Among them eosinophil cationic protein play important role in immune response to infections caused by bacteria, viruses, parasites and cytotoxicity to many human cell lines. Strategies to enhance the functional abilities of eosinophils by lineage specific differentiation of iPSC and enhanced expression of cytotoxic mediators by advanced genetic engineering approach provide important future application in such opportunistic infections.

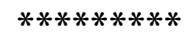

\section{Author Contributions}

Anubha Singh - Substantial contributions to conception and design, Acquisition of data, Analysis and interpretation of data, Drafting the article, Revising it critically for important intellectual content, Final approval of the version to be published

Deepak Kumar Singh - Analysis and interpretation of data, Revising it critically for important intellectual content, Final approval of the version to be published Usha Bhoria - Analysis and interpretation of data, Revising it critically for important intellectual content, Final approval of the version to be published

\section{Guarantor}

The corresponding author is the guarantor of submission.

\section{Conflict of Interest}

Authors declare no conflict of interest.

\section{Copyright}

(C) 2015 Anubha Singh et al. This article is distributed under the terms of Creative Commons Attribution License which permits unrestricted use, distribution and reproduction in any medium provided the original author(s) and original publisher are properly credited. Please see the copyright policy on the journal website for more information

\section{REFERENCES}

1. Bleakley M, Heimfeld S, Jones LA, et al. Engineering human peripheral blood stem cell grafts that are depleted of naïve $\mathrm{T}$ cells and retain functional pathogen-specific memory T cells. Biol Blood Marrow Transplant 2014 May;20(5):705-16.

2. Hanna J, Wernig M, Markoulaki S, et al. Treatment of sickle cell anemia mouse model with iPS cells generated from autologous skin. Science. 2007 Dec 21;318(5858):1920-3.

3. Lehrer R, Szklarek D, Barton A, Ganz T, Hamann KJ, Gleich GJ. Antibacterial properties of eosinophil major basic protein and eosinophil cationic protein. J Immunol 1989 Jun 15;142(12):4428-34.

4. Domachowske JB, Dyer KD, Adams AG, Leto TL, Rosenberg HF. Eosinophil cationic protein/RNase 3 is another RNase A-family ribonuclease with direct antiviral activity. Nucleic Acids Res 1998 Jul 15;26(14):3358-63.

5. McLaren DJ, McKean JR, Olsson I, Venges P, Kay AB. Morphological studies on the killing of schistosomula of Schistosoma mansoni by human eosinophil and neutrophil cationic proteins in vitro. Parasite Immunol 1981 Winter;3(4):359-73.

6. Hamann KJ, Barker RL, Loegering DA, Gleich GJ. Comparative toxicity of purified human eosinophil granule proteins for newborn larvae of Trichinella spiralis. J Parasitol 1987 Jun;73(3):523-9.

7. Molina HA, Kierszenbaum F, Hamann KJ, Gleich GJ. Toxic effects produced or mediated by human eosinophil granule components on Trypanosoma cruzi. Am J Trop Med Hyg 1988 Mar;38(2):327-34.

8. Singh A, Batra JK. Role of unique basic residues in cytotoxic, antibacterial and antiparasitic activities of human eosinophil cationic protein. Biol Chem 2011 Apr;392(4):337-46.

9. Benjamin P. Davis, Marc E. Rothenberg, Cancer Immunology Research, Eosinophils and Cancer, Cancer Immunol Res, 2014;2:1-8.

10. Hu M, Krause D, Greaves M, et al. Multilineage gene expression precedes commitment in the hemopoietic system. Genes Dev 1997 Mar 15;11(6):774-85.

11. Wernig M, Meissner A, Foreman R, et al. In vitro reprogramming of fibroblasts into a pluripotent EScell-like state. Nature 2007 Jul 19;448(7151):318-24.

12. Weinacht KG, Brauer PM, Felgentreff K, et al. The role of induced pluripotent stem cells in research and therapy of primary immunodeficiencies. Curr Opin Immunol 2012 Oct;24(5):617-24.

13. Hogan SP, Waddell A, Fulkerson PC. Eosinophils in infection and intestinal immunity. Curr Opin Gastroenterol 2013 Jan;29(1):7-14.

14. Differentiation of Human Embryonic and Induced Pluripotent Stem Cells into Blood Cells in Coculture with Murine Stromal Cells Human Embryonic and Induced Pluripotent Stem Cells Springer Protocols Handbooks 2012, pp. 321-35. 
Access full text article on other devices

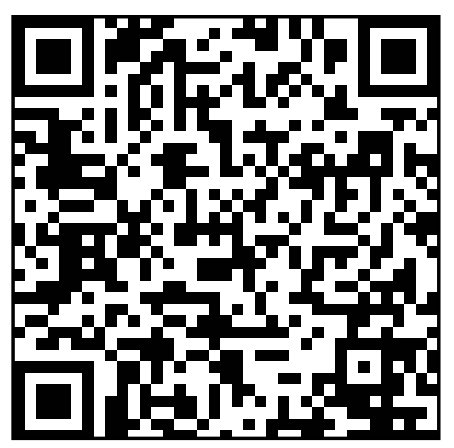

Access PDF of article on other devices

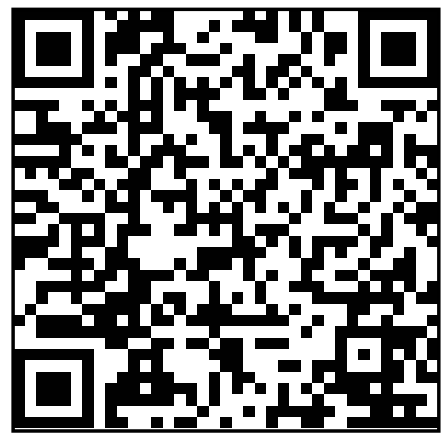

